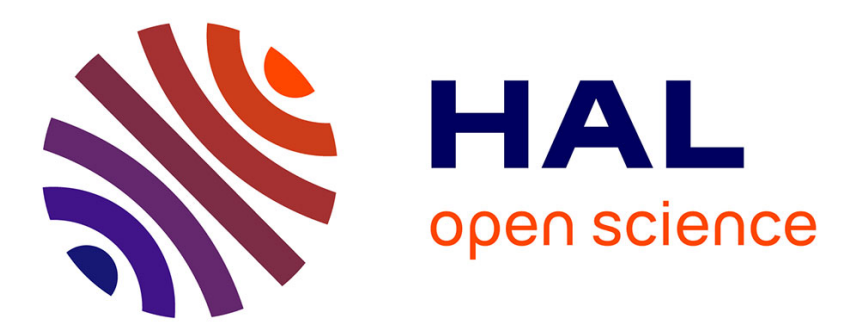

\title{
IL-4 mediated transcriptional regulation of human CYP2E1 by two independent signaling pathways
}

Jue Wang, Yin Hu, Jana Nekvindova, Magnus Ingelman-Sundberg, Etienne P.A. Neve

\section{- To cite this version:}

Jue Wang, Yin Hu, Jana Nekvindova, Magnus Ingelman-Sundberg, Etienne P.A. Neve. IL-4 mediated transcriptional regulation of human CYP2E1 by two independent signaling pathways. Biochemical Pharmacology, 2010, 80 (10), pp.1592. 10.1016/j.bcp.2010.08.005 . hal-00626228

\section{HAL Id: hal-00626228 \\ https://hal.science/hal-00626228}

Submitted on 24 Sep 2011

HAL is a multi-disciplinary open access archive for the deposit and dissemination of scientific research documents, whether they are published or not. The documents may come from teaching and research institutions in France or abroad, or from public or private research centers.
L'archive ouverte pluridisciplinaire HAL, est destinée au dépôt et à la diffusion de documents scientifiques de niveau recherche, publiés ou non, émanant des établissements d'enseignement et de recherche français ou étrangers, des laboratoires publics ou privés. 


\section{Accepted Manuscript}

Title: IL-4 mediated transcriptional regulation of human CYP2E1 by two independent signaling pathways

Authors: Jue Wang, Yin Hu, Jana Nekvindova, Magnus Ingelman-Sundberg, Etienne P.A. Neve

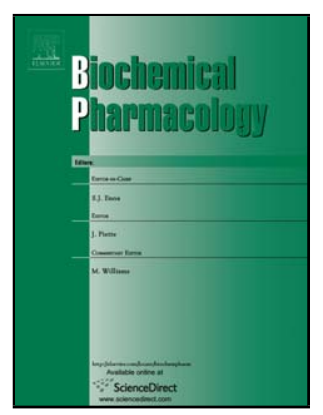

PII:

S0006-2952(10)00589-7

DOI: doi:10.1016/j.bcp.2010.08.005

Reference: BCP 10684

To appear in: $B C P$

Received date: 3-6-2010

Revised date: $5-8-2010$

Accepted date: $5-8-2010$

Please cite this article as: Wang $\mathrm{J}$, Hu Y, Nekvindova J, Ingelman-Sundberg M, Neve EPA, IL-4 mediated transcriptional regulation of human CYP2E1 by two independent signaling pathways, Biochemical Pharmacology (2010), doi:10.1016/j.bcp.2010.08.005

This is a PDF file of an unedited manuscript that has been accepted for publication. As a service to our customers we are providing this early version of the manuscript. The manuscript will undergo copyediting, typesetting, and review of the resulting proof before it is published in its final form. Please note that during the production process errors may be discovered which could affect the content, and all legal disclaimers that apply to the journal pertain. 


\section{IL-4 mediated transcriptional regulation of human CYP2E1 by two} independent signaling pathways

Running title: Mechanisms for IL-4 induced CYP2E1 expression

Jue Wang ${ }^{\mathrm{a}}$, Yin $\mathrm{Hu}^{\mathrm{b}}$, Jana Nekvindova ${ }^{\mathrm{a}}$, Magnus Ingelman-Sundberg ${ }^{\mathrm{a}}$ and Etienne P.A.

$\mathrm{Neve}^{\mathrm{a} *}$

${ }^{\text {a }}$ Section of Pharmacogenetics, Department of Physiology and Pharmacology,

Karolinska Institute, Stockholm, Sweden

${ }^{\mathrm{b}}$ Department of DMPK, AstraZeneca R\&D Södertälje, Sweden

* To whom correspondence should be addressed:

Etienne P.A. Neve. Section of Pharmacogenetics, Department of Physiology and

Pharmacology, Karolinska Institutet, Nanna Svartz väg 2, 17177 Stockholm, Sweden.

Tel: +468 52487711. Fax: +468337327. E-mail: etienne.neve@ki.se 


\begin{abstract}
Cytochrome P450 2E1 (CYP2E1), the alcohol-inducible member of the cytochrome P450 super family, plays an important role in both physiological and pathophysiological processes. The present study focused on the induction of human CYP2E1 transcription by the anti-inflammatory cytokine interleukin-4 (IL-4) in human hepatoma B16A2 cells and revealed that this regulation is mediated by two independent pathways. RNA interference and overexpression of STAT6, indicated that the JAKSTAT signaling pathway is involved in IL-4-dependent induction and mutagenesis revealed the presence of a STAT6 binding site in CYP2E1 proximal promoter region (583/-574-bp). However, inhibition of the JAK-STAT6 pathway using JAK1 siRNA constructs could only partially inhibit the induction of CYP2E1 promoter constructs indicating the presence of a second IL-4 responsive element. Indeed by using a series of truncated CYP2E1 promoter constructs a second more distal IL-4 responsive element (1604/-1428-bp) was identified, which was further shown to involve the activation of IRS1/2. This induction was dependent on the transcription factor NFATc1 as IL-4 induced CYP2E1 expression was altered by silencing or overexpressing NFATc1. A NFATc1 binding site was identified in the second distal IL-4 responsive element (1551/-1545-bp) by chromatin immunoprecipitation (ChIP) analysis. Finally simultaneous siRNA mediated down-regulation of both STAT6 and NFATc1 or mutation of both STAT6 and NFATc1 binding sites abolished the IL-4-dependent transcriptional induction of CYP2E1, demonstrating that both pathways are required for maximal activation. In conclusion, the present study indicates that the induction of CYP2E1 transcription by IL-4 is mediated through two independent parallel pathways, involving JAK-STAT6 and IRS1/2 and NFATc1.
\end{abstract}


Keywords: cytochrome P450 2E1, B16A2 cell line, interleukin-4, transcriptional activation, transcription factors

Abbreviations: ChIP, chromatin immunoprecipitation; CYP, cytochrome P450; Elk1, member of ETS oncogene family; FOXO1, forkhead box protein O1; IL-4, interleukin4; IL-4R, interleukin-4 receptor; IRS, insulin receptor substrate; JAK, janus protein kinase; MAPK, mitogen-acitvated protein kinase; NFATc1, nuclear factor of activated T-cells cytoplasmic 1; NF-кB, nuclear factor kappa B; PI3K, phosphoinositide-3-kinase; STAT6, signal transducer and activator of transcription 6. 


\section{Introduction}

Cytochrome P450 2E1 (CYP2E1), the ethanol-inducible form of cytochrome P450 (CYP), is an important phase I drug metabolizing enzyme since it plays a dual physiological role [1-4]. It contributes to the defense against the penetration of xenobiotics through its broad substrate specificity, allowing it to detoxify a wide variety of exogenous and endogenous compounds and also plays a role in gluconeogenesis. Paradoxically, it also metabolically activates biologically inert procarcinogens and converts nontoxic substrates to more toxic forms [5]. Furthermore, CYP2E1 is an effective generator of reactive oxygen species such as superoxide anion radicals, hydrogen peroxide and hydroxyl radicals, rendering it an important component in the process of oxidative stress $[2,3,6,7]$. As an important component of the adaptive system in the body, the expression of CYP2E1 is under strict regulation at different levels including transcriptional, post-transcriptional, translational, and post-translational [7-9]. CYP2E1 is induced by both acute and chronic alcohol treatment, as well as under a variety of physiological and pathophysiological conditions. For example, CYP2E1 expression is decreased during inflammation [10] and in alcoholic or non-alcoholic liver diseases [11, 12], and instead increased in diabetic patients [13, 14]. Cytokines, a group of secreted peptides and glycoproteins essential for cellular signaling, are shown to be key mediators in the regulation of CYPs during inflammation and infection [15].


necrosis factor (TNF) and interferon- $\alpha / \gamma(\mathrm{IFN}-\alpha / \gamma)$ were found to function in the regulation of specific CYPs during lipopolysaccharide (LPS)-induced inflammation. CYP2E1 is also regulated by cytokines both in human and rodent models [16, 17]. Similar to most other CYPs, CYP2E1 is down-regulated by pro-inflammatory cytokines, 
such as IL-1 $\beta$, IL-6 and TNF. Interestingly, Abdel-Razzak and coworkers demonstrated that in primary human hepatocytes interleukin-4 (IL-4), an anti-inflammatory cytokine, significantly up-regulates the expression of CYP2E1 as well as glutathione-Stransferases (GST), although it down-regulates most other P450s [16].

IL-4, mainly produced by $\mathrm{T}$ helper $\left(\mathrm{T}_{\mathrm{H}}\right) 2$ lymphocytes, is a key cytokine in the regulation of humoral immune response. It contributes to the activation, proliferation, and differentiation of B cells, the secretion of IgE by B lymphocytes, and the prevention of apoptosis in T lymphocytes. An essential biological activity of IL-4 in the development of allergic inflammation is the ability to drive the differentiation of naive T helper type $0\left(\mathrm{~T}_{\mathrm{H}} 0\right)$ lymphocytes into $\mathrm{T}_{\mathrm{H}} 2$ lymphocytes [18]. Its effect relies on signaling through a receptor complex consisting of the specific IL-4 receptor alpha chain (IL-4R $\alpha$ ) and the common gamma chain (IL-4R $\gamma \mathrm{c}$ ), resulting in a series of phosphorylation events mediated by receptor-associated kinases [18-21]. The IL-4R heterodimerization promotes the activation of Janus protein kinases (JAKs) that are constitutively associated with IL-4R $\alpha$ (JAK1) and the IL-4R $\gamma \mathrm{c}$ (JAK3).

Phosphorylation of a cassette of three closely spaced tyrosine residues in the cytoplasmic domain of IL-4R $\alpha$ initiated by activated JAKs enables the recruitment of the transcription factor signal transducer and activator of transcription 6 (STAT6). The tyrosine phosphorylation of STAT6 by JAKs leads to its dimerization and translocation to the nucleus, where it binds to specific cis-acting elements and activates the transcription of IL-4-responsive genes. In addition to the JAK-STAT pathway, the insulin receptor substrate (IRS) family of proteins is also involved in IL-4 signaling. Activation of IRS1/2 results in the activation of mitogen-activated protein kinase (MAPK) signaling cascades as well as the phosphoinositide-3-kinase (PI3K) pathway [21]. Furthermore IL-4-mediated induction of 3 3 -hydroxysteroid dehydrogenase/ $\Delta 5-\Delta 4$ 
isomerase as well as IL-4-induced proliferation and differentiation were shown to require simultaneous signaling via both STAT6 and IRS-2 [22, 23].

Previous studies showed that IL-4 induced expression of CYP2E1 is transcriptionally regulated and results in increased mRNA and protein levels in primary human hepatocytes and human hepatoma cells [16, 24]. In the present study using human CYP2E1 promoter constructs transfected into human B16A2 hepatoma cells, we identified two IL-4 responsive elements in the human CYP2E1 promoter, a proximal region (-519/-669-bp) and a more distal one (-1604/-1428-bp). Both regions were required for maximal IL-4 mediated induction of CYP2E1. Moreover siRNA experiments and mutagenesis of the CYP2E1promoter indicate that two well known IL4 signaling pathways are responsible for the IL-4 induction of CYP2E1, namely JAK1STAT6 and IRS1/2 involving the transcription factor nuclear factor of activated T-cells cytoplasmic 1 (NFATc1). 


\section{Material and methods}

\subsection{Plasmids}

Human CYP2E1 promoter constructs of various lengths were amplified by PCR and cloned in between $B g l \mathrm{II} / \mathrm{XhoI}$ restriction sites in pGL3-Basic vector (Promega, Madison, WI, USA), upstream of the Firefly luciferase reporter gene as previously described [25]. The lengths of CYP2E1 promoters varied from -519- to -3205-bp upstream of the transcriptional initiation codon. Reporter constructs generated 2E13205, 2E1-2651, 2E1-1604, 2E1-1428, 2E1-1231, 2E1-1009, 2E1-841, 2E1-669 and 2E1-519 contained CYP2E1 promoter regions up to -3205-bp, -2651-bp, -1604-bp, 1428-bp, -1231-bp, -1009-bp, -841-bp, -669-bp and -519-bp respectively. Mutations in the putative STAT6- and NFATc1-binding sites in the CYP2E1 promoter region were introduced by the QuikChange ${ }^{\circledR}$ Site-Directed Mutagenesis Kit (Stratagene, La Jolla, CA, USA) with mutagenesis primers shown in the Supplemental data (Table S1). Expression constructs for the transcription factors STAT6, NFATc1 and FOXO1, were kindly provided by Dr. Ulrike Schindler (Amgen, Thousand Oaks, CA, USA), Dr. Edgar Serfling (Wurzburg University, Wurzburg, Germany) and Dr. Anjana Rao (Harvard Medical School, Boston, MA, USA) respectively. All constructs used in the present study were confirmed by DNA sequencing.

\subsection{RNA interference assay}

Potential siRNA sequences were designed using online siRNA selection algorithm (https://www.genscript.com/ssl-bin/app/rnai), DNA oligonucleotides (Supplemental data, Table S1) were ordered from Invitrogen (Carlsbad, CA, USA) as specified in GeneBuster users manual (Genordia AB, Sundbyberg, Sweden), annealed 
and cloned into GeneBuster vector according to the manufacturer's instruction.

Resulting siRNA expression constructs were transfected into B16A2 cells alone or together with CYP2E1 reporter constructs. At least two efficient siRNAs were used to target each gene to exclude the potential off-target effects of RNA interference.

\subsection{Cell culture, transient transfection and reporter gene analysis}

The human hepatoma cell line B16A2 [26] was maintained in William's E medium (Invitrogen, Carlsbad, $\mathrm{CA}, \mathrm{USA}$ ) at $37^{\circ} \mathrm{C}$ in an incubator with $5 \% \mathrm{CO}_{2}$, supplemented with $1 \mu \mathrm{g} / \mathrm{ml}$ insulin, $0.25 \mu \mathrm{g} / \mathrm{ml}$ hydrocortisone, $5 \% \mathrm{FBS}$, penicillin (100 IU/mL) and streptomycin $(100 \mu \mathrm{g} / \mathrm{mL})$ (Invitrogen). B16A2 cells were grown to confluence in 12 well clusters (after 1 week of culture following being split 1/6) and 4 weeks after reaching confluence were co-transfected with $1 \mu \mathrm{g}$ of CYP2E1 reporter constructs and 50 ng pRL-TK (Renilla luciferase) control plasmid (Promega) using the DMRIE-C transfection reagent (Invitrogen).with weeks after reaching confluence.

Where indicated, cells were also co-transfected with siRNA expression vectors $(0.5 \mu \mathrm{g})$, expression vectors for the transcription factors STAT6, NFATc1, Elk1, NF- $\kappa$ B and FOXO1 $(0.5 \mu \mathrm{g})$ or their corresponding empty control vectors. Twenty-four hours posttransfection cells were either left untreated or treated with $60 \mathrm{U} / \mathrm{ml}$ human recombinant IL-4 (Sigma-Aldrich, St. Louis, MO, USA). The luciferase activity of both the CYP2E1 reporter gene (Firefly luciferase) and the internal control plasmid pRL-TK (Renilla luciferase) was measured in a TD-20/20 luminometer (Turner Designs, Sunnyvale, CA, USA) with the Dual Luciferase reporter assay system (Promega). 


\subsection{Northern blot assay}

Total RNA was prepared from IL-4 treated and untreated B16A2 cells (4 weeks post-confluence) using single-step RNA isolation by acid guanidium thiocyanatephenol-chloroform extraction method described by Chomczynski and Sacchi [27]. Northern blot assays were performed as previously described except that $20 \mu \mathrm{g}$ of total RNA was loaded per lane [28].

\subsection{Real time PCR}

Total RNA was isolated from treated and untreated B16A2 cells with the RNeasy mini kit (Qiagen, Valencia, CA, USA). The mRNA levels were quantified by real time PCR using SYBR green PCR master mix (Applied Biosystems, Foster City, CA, USA) on a smart cycler (Cepheid, Sunnyvale, CA, USA) as described [29]. The mRNA levels were normalized against $\beta$-actin. Primers used are listed in Supplemental data (Table S1).

\subsection{Chromatin immunoprecipitation (ChIP) assay}

ChIP assays were performed using the ChIP assay kit from Upstate according to the manufacturer's instructions (Upstate, Temecula, CA, USA). B16A2 cells were grown confluent in $10 \mathrm{~cm}$ cell culture dishes for 4 weeks, transfected with 2E1-2651 and $24 \mathrm{~h}$ post-transfection cells were either left untreated or treated with $60 \mathrm{U} / \mathrm{ml}$ IL-4. Cells were processed for ChIP analysis $24 \mathrm{~h}$ after the addition of IL-4. DNA-protein complexes were cross-linked by the addition of $1 \%$ formaldehyde directly to the culture medium after which cells were washed and lysed in SDS Lysis buffer supplemented with protease and phosphatase inhibitors (Roche) and processed as suggested by the 
manufacturer. DNA-protein complexes were immunoprecipitated with a mouse monoclonal anti-NFATc1 antibody and normal mouse IgG (both from Santa Cruz Biotechnology, Santa Cruz, CA, USA). Immunoprecipitated DNA was eluted, purified and amplified by PCR for $25-30$ cycles in the presence of $1.5 \mathrm{mM} \mathrm{MgCl}_{2}, 200 \mu \mathrm{M}$ dNTPs and 10 pmol of primers (Supplemental data, Table S1). The PCR primer set was designed to yield a 160-bp product that encompassed the region containing the NFATc1 responsive element. $10 \%$ of the precipitated DNA was used for each PCR. PCR products were separated by electrophoresis on $2 \%$ agarose gels and visualized with ethidium bromide. 


\section{Results}

\subsection{IL-4 induces CYP2E1 expression}

In a previous study we demonstrated that when grown confluent for 5 weeks B16A2 cells display a 7-fold increase in CYP2E1 mRNA expression as compared to non-confluent cells, although these levels still were low compared to human liver [30]. Others demonstrated that IL-4 treatment increased both CYP2E1 mRNA and protein levels in primary human hepatocytes cultures and also, in a concentration dependent manner, in confluent grown B16A2 cells $[16,24]$. To verify the induction in our experimental system, 4 week confluent B16A2 cells were treated with IL-4 (Fig. 1). As expected in untreated control cells low levels of CYP2E1 mRNA could be detected, which increased 2.5-fold $4 \mathrm{hr}$ after the addition of IL-4 (Fig. 1) and remained stable up to $24 \mathrm{hrs}$ after the addition of IL-4 (data not shown). These results confirm that the induction pathway mediated by IL-4 is functional in our cultured B16A2 cells and possibly is regulated at the transcriptional level as was also previously demonstrated [24].

In order to elucidate the regions in the human CYP2E1 promoter responsible for the transcriptional induction of CYP2E1 by IL-4, a set of CYP2E1 promoter constructs were generated (Fig. 2). Confluent B16A2 cells were transfected with these CYP2E1 reporter constructs of variable length and promoter activities were determined after IL4 treatment. IL-4 treatment resulted in a 2.5 - to 6-fold induction of the luciferase activity depending on the length of CYP2E1 promoter as compared to untreated cells, with the exception of the shortest construct, 2E1-519, which was unresponsive to IL-4. The data indicate that two regions in the CYP2E1 promoter contained IL-4 responsive elements, a proximal region between -669- and -519-bp upstream of the transcriptional 


\subsection{JAK1-STAT6 signaling pathway}

Although the JAK1-STAT6 signaling pathway is well-known and specifically activated by IL-4 [21], the direct involvement of STAT6 in IL-4-mediated induction of CYP2E1 has not been demonstrated, despite the presence of several STAT6 binding sites in the proximal IL-4 responsive element. We initially studied the involvement of JAK1-STAT6 signaling in IL-4-mediated induction of CYP2E1 using silencing and overexpression of several of the components involved in this signaling pathway. First we observed that IL-4 treatment resulted in a 2.8 -fold increase in JAK1 mRNA levels, which could be effectively prevented by 2 different JAK1-specific siRNA constructs down to or below control levels (Fig. 3A). Silencing of JAK1 expression using either one of these siRNAs prevented the IL-4-induced transcription of CYP2E1 promoter construct 2E1-669 that contained only the proximal IL-4 responsive element (Fig. 3B, left). When using the longer reporter construct, 2E1-1604 that contained both the proximal and distal IL-4 responsive element, the JAK1 siRNAs significantly inhibited, but not completely abolished, the IL-4-induced CYP2E1 transcription (Fig. 3B, right). This suggested that JAK1 is involved in the IL-4 transcriptional induction of CYP2E1 and that JAK1 mediated its effect through the proximal IL-4 responsive element. Moreover, since JAK1 down-regulation was not able to completely prevent the IL-4 
response in the presence of both the proximal and distal IL-4 responsive element suggested that the IL-4 response via the distal element, most likely, is mediated by a second signaling pathway.

STAT6 is a well-known downstream component of JAK1 after IL-4 stimulation and we therefore studied its involvement in the IL-4 induction of CYP2E1. Coexpression of STAT6 (in the absence of IL-4) with 2E1-1428, containing only the proximal IL-4 responsive element, or 2E1-1604, containing both the proximal and distal responsive elements, increased the promoter activity about 2-fold as compared to 2E1-519 that lacks both IL-4 responsive elements (Fig. 4A). Because no further induction by STAT6 overexpression was observed in the presence of the distal element it was concluded that the STAT6 binding site is located in the proximal promoter region. Four potential STAT6 binding sites (STAT6-1: -42-/-34-bp; STAT6-2: -583-/574-bp; STAT6-3: -623-/-615-bp and STAT6-4: -699-/-690-bp) were identified in the proximal CYP2E1 promoter that exhibited the STAT6 consensus $\left(\mathrm{TTCN}_{3-6} \mathrm{GAA}\right)$. These 4 sites were mutated individually in the reporter construct 2E1-841 using the mutagenesis primers listed in Supplemental data (Table S1). Wild-type 2E1-841 and the mutants $\triangle$ STAT6-1 to -4 were transfected into confluent B16A2 cells and transfected cells were treated with IL-4 or left untreated (Fig. 4B). With the exception of the mutant $\Delta$ STAT6-2 promoter construct, which was unresponsive to IL-4 treatment, the wild-type construct and the mutants $\Delta$ STAT6-1, $\Delta$ STAT6-3 and $\Delta$ STAT6-4 all displayed approximately a 2-fold increase after IL-4 treatment. These data show that the IL-4 responsive element for the JAK1-STAT6 pathway is located between -583and -574-bp in the CYP2E1 promoter and that mutation of this site abolishes the IL-4 response. 


\subsection{IRS signaling}

The data presented above suggests that the proximal IL-4 responsive element is activated through JAK1-STAT6 signaling, while the distal IL-4 responsive element is not. Because IL-4 can signal via the IL-4R not only through the JAK-STAT pathway but also through activation of IRS, we decided to investigate the involvement of IRS in the IL-4 induction of CYP2E1. Indeed both IRS1 and IRS2 mRNA levels were significantly up-regulated after IL-4 treatment of confluent B16A2 cells (Fig. 5A). Silencing of IRS1 or IRS2 alone did not significantly inhibit the IL-4-induced reporter gene expression when using 2E1-1606 that contains both the proximal and distal IL-4 responsive element (Fig. 5B), despite the fact that both siIRS1-2 and siIRS2-2 effectively down-regulated their respective targets (Fig. 5A). However, when both IRS1 and IRS2 were silenced simultaneously, the IL-4-induced CYP2E1 reporter activity could be significantly inhibited by about $40 \%$ (Fig. 5B).

We next analyzed the sequence of the distal IL-4 responsive element between 1604- and -1428-bp upstream of CYP2E1 initiation codon using the TESS (Transcriptional Element Search System) program for the presence of putative transcription factor binding sites [31]. Eight of the transcription factors identified, were chosen for further evaluation since they have been shown to be involved in IL-4 response (KEGG, SPAD and ProteinLounge Pathway databases). First we determined the fold induction at the mRNA level of these eight transcription factors after IL-4 treatment of B16A2 cells (Fig. 6A) and observed that 4 of them, more specifically NF$\kappa \mathrm{B}$ (nuclear factor kappa B), Elk1 (member of ETS oncogene family), NFATc1 (nuclear factor of activated T-cells cytoplasmic 1) and FOXO1 (forkhead box protein O1) were induced 2- to 5-fold by IL-4 treatment. In order to establish the direct involvement of these transcription factors in the IL-4-induced CYP2E1 expression, we 
generated siRNA expression vectors to effectively silence these transcription factors (Supplemental data, Fig. S1). Silencing of NFATc1 significantly attenuated the IL-4 induced CYP2E1 expression, while down-regulation of the other factors, NF-kB, Elk1 and FOXO1 had no significant effect (Fig. 6B). To corroborate the involvement of NFATc1 in the IL-4-induced CYP2E1 expression, B16A2 cells were transfected with the CYP2E1 promoter construct containing the proximal and distal IL-4 responsive element (2E1-1604) in the presence and absence of a NFATc1 expression plasmid (Fig. 6C). Indeed co-expression of 2E1-1604 and NFATc1 resulted in a 2-fold increase in promoter activity as compared to 2E1-1604 alone. In contrast, co-expression with FOXO1 did not alter the promoter activity as was expected from the lack of effect from the down regulation of FOXO1, thereby excluding a role of FOXO1 in the IL-4dependent induction of CYP2E1 expression. To test whether NFATc1 could directly interact with the distal IL-4 responsive element in the CYP2E1 promoter, we performed chromatin immunoprecipitation (ChIP) analysis using primers for this specific region containing the putative NFATc1 binding site (Supplemental data, Table S1). In B16A2 transfected with 2E1-2651, NFATc1 was found to interact with the distal IL-4 responsive element in the CYP2E1 promoter (Fig. 6D). Stimulation of these transfected cells with IL-4 however did not enhance but rather decreased the binding of NFATc1 to the CYP2E1 promoter, indicating that in control confluent B16A2 cells, which contain low CYP2E1 levels, NFATc1 is already constitutively associated with the CYP2E1 promoter. Moreover we also could detect a weak interaction of NFATc1 with the endogenous CYP2E1 promoter in non-transfected cells (Supplemental data, Fig. S2). In contrast we did observe an IL-4 dependent interaction with the distal IL-4 responsive element containing the NFATc1 binding site using electrophoretic mobility shift assay and nuclear extracts isolated from control confluent and IL-4 treated confluent B16A2 
cells (Supplemental data, Fig. S3). The reason for the discrepancy between the ChIP and EMSA and the slight decrease in NFATc1 binding to the CYP2E1 promoter in the presence of IL-4 observed by ChIP is at present unclear, but involvement of additional transcription factors can at this point not be excluded.

\subsection{Both STAT6 and NFATc1 are required for efficient induction by IL-4}

To further confirm the involvement of STAT6 and NFATc1, the activity of both transfection factors was inhibited by using either RNA interference or by mutation of the transcription factor binding sites (Fig. 7). Mutation of either the STAT6 ( $\triangle$ STAT6) or NFATc1 ( $\triangle$ NFATc1) binding site (schematically shown at the bottom of Fig. 7) resulted only in a slight (about 30-35\%) but significant inhibition of the IL-4-mediated induction of CYP2E1 promoter activity. When both sites were mutated simultaneously ( $\triangle$ STAT6 $+\triangle$ NFATC1) the effect of IL-4 on CYP2E1 promoter activity was essentially eliminated, confirming that efficient IL-4 induction of CYP2E1 expression required both STAT6 and NFATc1 interaction with the CYP2E1 promoter. In addition, siRNAmediated down-regulation of both transcription factors STAT6 and NFATc1 also abolished the IL-4 induction of CYP2E1 promoter activity (Fig. 7), confirming once more their involvement. Thus, our data strongly indicate that the induction of CYP2E1 transcription by IL- 4 treatment is mediated by two independent pathways, involving the transcription factors STAT6 and NFATc1. 


\section{Discussion}

In the present study we provide evidence that IL-4 induction of CYP2E1 in the human hepatoma cell line B16A2 is mediated by two classical IL-4 signaling pathways, involving the JAK1-STAT6 pathway and activation of IRS1/2 and NFATc1. Using different length promoter constructs combined with siRNA constructs against JAK1, STAT6, IRS1/2 and NFATc1 we could show that IL-4 mediates its effect through two distinct regions in the human CYP2E1 promoter: a proximal IL-4 responsive element (669/-519-bp) activated by the JAK1-STAT6 pathway and a more distal site (-1604/1428-bp) activated by IRS1/2. Finally, we showed using mutagenesis and siRNA constructs that these two IL-4 responsive elements are activated by different transcription factors, the proximal IL-4 responsive element (-583/-574-bp) by STAT6 and the distal IL-4 responsive element (-1551/-1545-bp) by NFATc1. Both pathways appear to operate in parallel and are required for maximal response since in order to prevent the IL-4-dependent CYP2E1 induction, we needed to block both pathways simultaneously. These findings are schematically summarized in Fig. 9, together with the factors and sites that were, in the present study, shown to be important for the IL-4 induction of CYP2E1. In contrast to the well characterized JAK-STAT pathway, at present it is unclear how activation of IRS could lead to the activation of NFATc1. Our results clearly show that both IRS and NFATc1 mediate their effect on CYP2E1 transcription via the distal IL-4 responsive element. In a recent report it was shown that the expression of NFATc1 was regulated by the ERK signaling pathway as it was shown that NFATc1 expression was inhibited by the MEK inhibitor U0126 [32]. We could partially inhibit the IL-4 induction of CYP2E1 with the same MEK inhibitor (U0126), indicating the involvement of this protein kinase (Supplemental data, Fig. S4). 
No effect could be observed using a p38-MAPK inhibitor (SB203580) or a PI3K

inhibitor (wortmannin), suggesting these two protein kinases are not involved in the IL4-induced expression of CYP2E1 (Supplemental data, Fig. S4). Interestingly, IL-4 mediated stimulation of IRS leads to the activation of MEK/ERK pathway [22], which raises the possibility that IL-4 is able to activate CYP2E1 expression via activation of IRS and subsequently MEK resulting in activation of NFATc1.

Confluent grown B16A2 cells start expressing several liver specific genes including CYP2E1 both at the mRNA and protein level $[24,30]$. Low amounts of CYP2E1 protein could already be observed after 1-2 weeks of confluence and gradually increased with time of confluence (data not shown). Stimulation of confluent B16A2 cells with the cytokine IL-4 resulted in an additional increase in CYP2E1 mRNA and protein expression (present study) [24], a phenomenon that was also observed in primary human hepatocytes [16]. Although in our study we present evidence for the involvement of STAT6, a well-known downstream target of IL-4 signaling, a previous study suggested that IL-4-induced CYP2E1 transcription independent of STAT6, and instead proposed the involvement of protein kinase C [24]. In the present study a STAT6 binding site in the CYP2E1 promoter is identified by mutagenesis and shown to be involved in the IL-4 induced expression of CYP2E1. The same region in the CYP2E1 promoter was identified in another study to be important for IL-4-induced CYP2E1 expression, but electrophoretic mobility shift assays failed to show STAT6 binding and instead AP1 and NFAT were shown to bind to this IL-4 responsive element [33]. These results are in contrast to ours, as IL-4 induction of CYP2E1 could be completely prevented after mutagenesis of the STAT6 binding site present in the proximal IL-4 responsive element ( $\triangle$ STAT6-2) when using the short promoter 
constructs that lacked the second more distal IL-4 response element. The reason for this inconsistency is at present not clear.

Confluent grown B16A2 cells already do express low levels of CYP2E1 [24, 30], which suggests that during confluence the CYP2E1 promoter becomes activated by as yet unknown molecular mechanisms. We observed a weak interaction of NFATc1 with the endogenous CYP2E1 promoter in the absence of IL-4 (Supplemental data, Fig. S2), suggesting that NFATc1 is associated with the CYP2E1 promoter and is able to regulate its activity. We propose that prolonged confluence induces certain signaling cascades ultimately leading to activation and binding of NFATc1 and subsequent transcriptional activation of CYP2E1. In rat hepatocytes, low amounts of insulin induced CYP2E1 expression, while higher concentrations, led to suppression [34]. B16A2 cells are grown confluent in the presence of low amounts of insulin that possibly may result in activation of IRS signaling and ultimately lead to transcriptional activation of CYP2E1.

The regulation of CYP2E1 is complex and is know to occur at the transcriptional, translational, and posttranscriptional level [7, 9], although transcriptional activation of CYP2E1 remains poorly understood. Immediately after birth CYP2E1 becomes transcriptionally activated, and was accompanied by demethylation at cytosine residues located in the 5'-flanking region of the rat gene [35]. Several transcription factors have been shown to interact with and activate the CYP2E1 promoter as was shown for HNF-1 $\alpha$ in rat [17, 36], Sp1 and NF-KB in rabbit [37] and STAT5 (tonicity response element binding protein) in human [38]. Furthermore also in human, a far upstream enhancer site was identified and shown to be regulated by the transcription factors GATA4 and NR5A2 (fetoprotein transcription factor) [39]. IL-1 $\alpha$ was shown to up-regulate CYP2E1 promoter activity in rabbit through activation of 
Sp1, while IL-4 had no significant effect on the promoter activity in rabbit [37]. In contrast, in rats, IL-1 $\alpha$ treatment down-regulated CYP2E1 mRNA levels [40] and comparison of the rabbit rat and human CYP2E1 promoter revealed that the Sp1 site found in rabbit is not conserved in the rat and human promoter [37]. This illustrates that regulation of CYP2E1 transcription is highly species dependent and that the same transcription factor or stimulus can have opposite effects depending on the species studied as illustrated by IL- $1 \alpha$ and Sp1.

In conclusion, we demonstrate that the regulation of CYP2E1 by IL-4 was mediated by two independent pathways, the JAK1-STAT6 pathway and activation of IRS1/2. These two pathways were independent from each other, both requiring specific transcriptional factors as well as distinct responsive sites in the CYP2E1 promoter, a proximal site involving STAT6 and a distal one dependent on NFATc1. The regulation is under the control of a complex set of factors and constitutes part of the signaling transduction network. In addition we demonstrate that the human hepatoma cell line B16A2 when grown confluent, can be a useful tool for studying the regulation of endogenous hepatic proteins.

Acknowledgements: This project was supported by The Swedish Research Council. The authors would like to thank Dr. Ulrike Schindler (Amgen, Thousand Oaks, CA, USA), Dr. Edgar Serfling (Wurzburg University, Wurzburg, Germany) and Dr. Anjana Rao (Harvard Medical School, Boston, MA, USA) for sharing expression constructs for STAT6, NFATc1 and FOXO1 respectively. 


\section{References}

[1] Badger TM, Ronis MJ, Seitz HK, Albano E, Ingelman-Sundberg M, Lieber CS. Alcohol metabolism: role in toxicity and carcinogenesis. Alcohol Clin Exp Res $2003 ; 27: 336-47$.

[2] Caro AA, Cederbaum AI. Oxidative stress, toxicology, and pharmacology of CYP2E1. Annu Rev Pharmacol Toxicol 2004;44:27-42.

[3] Gonzalez FJ. Role of cytochromes P450 in chemical toxicity and oxidative stress: studies with CYP2E1. Mutat Res 2005;569:101-10.

[4] Lieber CS. The discovery of the microsomal ethanol oxidizing system and its physiologic and pathologic role. Drug Metab Rev 2004;36:511-29.

[5] Lieber CS. Cytochrome P-4502E1: its physiological and pathological role. Physiol Rev 1997;77:517-44.

[6] Cederbaum AI. Iron and CYP2E1-dependent oxidative stress and toxicity. Alcohol 2003;30:115-20.

[7] Ingelman-Sundberg M, Ronis MJ, Lindros KO, Eliasson E, Zhukov A. Ethanolinducible cytochrome P4502E1: regulation, enzymology and molecular biology. Alcohol Alcohol Suppl 1994;2:131-9.

[8] Aguiar M, Masse R, Gibbs BF. Regulation of cytochrome P450 by posttranslational modification. Drug Metab Rev 2005;37:379-404.

[9] Novak RF, Woodcroft KJ. The alcohol-inducible form of cytochrome P450 (CYP 2E1): role in toxicology and regulation of expression. Arch Pharm Res $2000 ; 23: 267-82$.

[10] Projean D, Dautrey S, Vu HK, Groblewski T, Brazier JL, Ducharme J. Selective downregulation of hepatic cytochrome P450 expression and activity in a rat model of inflammatory pain. Pharm Res 2005;22:62-70. 
[11] Lieber CS. CYP2E1: from ASH to NASH. Hepatol Res 2004;28:1-11.

[12] Nagy LE. Molecular aspects of alcohol metabolism: transcription factors involved in early ethanol-induced liver injury. Annu Rev Nutr 2004;24:55-78.

[13] Haufroid V, Ligocka D, Wallemacq P, Lison D, Horsmans Y. Comparison of cytochrome P4502E1 (CYP2E1) activity and hepatic and lymphocyte mRNA expression in patients with chronic hepatitis C. Toxicol Lett 2005;155:171-7.

[14] Wang Z, Hall SD, Maya JF, Li L, Asghar A, Gorski JC. Diabetes mellitus increases the in vivo activity of cytochrome P450 2E1 in humans. Br J Clin Pharmacol 2003;55:77-85.

[15] Aitken AE, Richardson TA, Morgan ET. Regulation of drug-metabolizing enzymes and transporters in inflammation. Annu Rev Pharmacol Toxicol 2006;46:123-49.

[16] Abdel-Razzak Z, Loyer P, Fautrel A, Gautier JC, Corcos L, Turlin B, et al. Cytokines down-regulate expression of major cytochrome P-450 enzymes in adult human hepatocytes in primary culture. Mol Pharmacol 1993;44:707-15.

[17] Hakkola J, Hu Y, Ingelman-Sundberg M. Mechanisms of down-regulation of CYP2E1 expression by inflammatory cytokines in rat hepatoma cells. J Pharmacol Exp Ther 2003;304:1048-54.

[18] Steinke JW, Borish L. Th2 cytokines and asthma. Interleukin-4: its role in the pathogenesis of asthma, and targeting it for asthma treatment with interleukin-4 receptor antagonists. Respir Res 2001;2:66-70.

[19] Boothby M, Mora AL, Stephenson LM. Lymphokine-dependent proliferation of T-lymphoid cells: regulated responsiveness and role in vivo. Crit Rev Immunol 2001;21:487-522. 
[20] Jiang H, Harris MB, Rothman P. IL-4/IL-13 signaling beyond JAK/STAT. J Allergy Clin Immunol 2000;105:1063-70.

[21] Nelms K, Keegan AD, Zamorano J, Ryan JJ, Paul WE. The IL-4 receptor: signaling mechanisms and biologic functions. Annu Rev Immunol 1999;17:70138.

[22] Gingras S, Cote S, Simard J. Multiple signal transduction pathways mediate interleukin-4-induced 3beta-hydroxysteroid dehydrogenase/Delta5-Delta4 isomerase in normal and tumoral target tissues. J Steroid Biochem Mol Biol 2001;76:213-25.

[23] Wurster AL, Withers DJ, Uchida T, White MF, Grusby MJ. Stat6 and IRS-2 cooperate in interleukin 4 (IL-4)-induced proliferation and differentiation but are dispensable for IL-4-dependent rescue from apoptosis. Mol Cell Biol 2002;22:117-26.

[24] Lagadic-Gossmann D, Lerche C, Rissel M, Joannard F, Galisteo M, Guillouzo A, et al. The induction of the human hepatic CYP2E1 gene by interleukin 4 is transcriptional and regulated by protein kinase C. Cell Biol Toxicol 2000;16:221-33.

[25] Hu Y, Hakkola J, Oscarson M, Ingelman-Sundberg M. Structural and functional characterization of the 5'-flanking region of the rat and human cytochrome P450 2E1 genes: identification of a polymorphic repeat in the human gene. Biochem Biophys Res Commun 1999;263:286-93.

[26] Le Jossic C, Glaise D, Corcos L, Diot C, Dezier JF, Fautrel A, et al. transActing factors, detoxication enzymes and hepatitis B virus replication in a novel set of human hepatoma cell lines. Eur J Biochem 1996;238:400-9. 
[27] Chomczynski P, Sacchi N. Single-step method of RNA isolation by acid guanidinium thiocyanate-phenol-chloroform extraction. Anal Biochem 1987;162:156-9.

[28] Hu Y, Mishin V, Johansson I, von Bahr C, Cross A, Ronis MJ, et al. Chlormethiazole as an efficient inhibitor of cytochrome P450 2E1 expression in rat liver. J Pharmacol Exp Ther 1994;269:1286-91.

[29] Wang J, Pitarque M, Ingelman-Sundberg M. 3'-UTR polymorphism in the human CYP2A6 gene affects mRNA stability and enzyme expression. Biochem Biophys Res Commun 2006;340:491-7.

[30] Butura A, Johansson I, Nilsson K, Warngard L, Ingelman-Sundberg M, Schuppe-Koistinen I. Differentiation of human hepatoma cells during confluence as revealed by gene expression profiling. Biochem Pharmacol 2004;67:1249-58.

[31] Schug J. Using TESS to predict transcription factor binding sites in DNA sequence. Curr Protoc Bioinformatics 2008;Chapter 2:Unit 26.

[32] Lee MS, Kim HS, Yeon JT, Choi SW, Chun CH, Kwak HB, et al. GM-CSF regulates fusion of mononuclear osteoclasts into bone-resorbing osteoclasts by activating the Ras/ERK pathway. J Immunol 2009;183:3390-9.

[33] Abdel-Razzak Z, Garlatti M, Aggerbeck M, Barouki R. Determination of interleukin-4-responsive region in the human cytochrome P450 2E1 gene promoter. Biochem Pharmacol 2004;68:1371-81.

[34] Woodcroft KJ, Hafner MS, Novak RF. Insulin signaling in the transcriptional and posttranscriptional regulation of CYP2E1 expression. Hepatology 2002;35:263-73. 
[35] Ueno T, Gonzalez FJ. Transcriptional control of the rat hepatic CYP2E1 gene. Mol Cell Biol 1990;10:4495-505.

[36] Liu SY, Gonzalez FJ. Role of the liver-enriched transcription factor HNF-1 alpha in expression of the CYP2E1 gene. DNA Cell Biol 1995;14:285-93.

[37] Peng HM, Coon MJ. Promoter function and the role of cytokines in the transcriptional regulation of rabbit CYP2E1 and CYP2E2. Arch Biochem Biophys 2000;382:129-37.

[38] Ito T, Asakura K, Tougou K, Fukuda T, Kubota R, Nonen S, et al. Regulation of cytochrome P450 2E1 under hypertonic environment through TonEBP in human hepatocytes. Mol Pharmacol 2007;72:173-81.

[39] Shadley JD, Divakaran K, Munson K, Hines RN, Douglas K, McCarver DG. Identification and functional analysis of a novel human CYP2E1 far upstream enhancer. Mol Pharmacol 2007;71:1630-9.

[40] Sindhu RK, Sakai H, Okamoto T, Kikkawa Y. Differential effect of interleukin1 alpha on rat hepatic cytochrome $\mathrm{P} 450$ monooxygenases. Toxicology 1996;114:37-46. 


\section{Figure legends}

Figure 1. IL-4 induces CYP2E1 mRNA expression in B16A2 cells. Confluent B16A2 cells were treated with $60 \mathrm{U} / \mathrm{ml} \mathrm{IL}-4$ for $4 \mathrm{hrs}$ and CYP2E1 mRNA levels were determined by Northern blot analysis (upper panel) and quantified by densitometry (lower panel). mRNA levels are expressed relative to untreated (control) cells and normalized to $\beta$-actin levels. Results are mean \pm S.D. of three independent experiments. $* *, \mathrm{P}<0.01$

Figure 2. Identification of the IL-4 responsive elements in the human CYP2E1 promoter. Different lengths of the CYP2E1 promoter were cloned upstream the luciferase reporter gene and transfected in confluent B16A2 cells. Transfected cells were either left untreated or treated with IL-4 for 24 hours, after which the luciferase activity was measured. Results are expressed as the fold induction of IL-4 treated over untreated control cells and normalized against Renilla luciferase activity. The values represent the average of at least 3 experiments. A schematic representation of the different lengths of the CYP2E1 promoter constructs is shown on the left side of the figure.

Figure 3. IL-4-mediated up-regulation of CYP2E1 is mediated through JAK1. (A) IL-4 treatment of confluent B16A2 cells induced the expression of JAK1 mRNA levels as determined by real-time PCR. Total RNA was prepared from B16A2 cells treated with IL-4 or left untreated and JAK1 mRNA levels were determined and normalized to $\beta$ actin levels. Two siRNA expression plasmids harboring the sequence targeting JAK1 (siJAK1-1 and siJAK1-2) were transfected prior to IL-4 treatment. Significant 
inhibition of JAK1 mRNA level was observed with both siRNA's. (B) IL-4 induced transcription of CYP2E1 is inhibited by down-regulation of JAK1. The two siRNA expression constructs targeting JAK1 were co-transfected with the CYP2E1 promoter constructs 2E1-669 (left-side) and 2E1-1604 (right-side). Twenty-four hours posttransfection cells were treated with IL-4 or left untreated for an additional $24 \mathrm{hrs}$ and luciferase activity was measured. Results are mean \pm S.D. of three individual experiments. *, $\mathrm{P}<0.05 ; * *, \mathrm{P}<0.01$.

Figure 4. IL-4 induced CYP2E1 transcription is mediated through STAT6. (A) A STAT6 expression plasmid was co-transfected with CYP2E1 reporter plasmids of different lengths, 2E1-519, 2E1-1428 and 2E1-1609, and 48 hrs post-transfection luciferase activity was determined. (B) Four putative binding sites for STAT6 in the CYP2E1 promoter were mutated as described in Materials and Methods. The STAT6 mutants ( $\triangle$ STAT6-1 to $\triangle$ STAT6-4) together with the wild-type CYP2E1 (wt 2E1) reporter construct were co-transfected with the STAT6 expression plasmid, 24 hrs posttransfection cells were treated with IL-4 (filled bars) or left untreated (empty bars) and after an additional $24 \mathrm{hrs}$ luciferase activity was determined. Results are mean \pm S.D. of three individual experiments. *, $\mathrm{P}<0.05$.

Figure 5. IL-4-induced expression of CYP2E1 is mediated through the activation of IRS1/2. (A) mRNA levels of IRS1 and IRS2 were measured in confluent control and IL-4 treated B16A2 cells. Two different siRNA's targeting IRS1 (siIRS1-1 and -2) or IRS2 (siIRS2-1 and -2) were designed and shown to effectively knockdown the mRNA levels of their respective targets after IL-4 treatment. (B) Simultaneous down-regulation of IRS1 and IRS2 attenuates the IL-4 response. IRS1-2 and IRS2-2 siRNA targeting 
constructs were individually or together co-transfected with the CYP2E1 promoter construct 2E1-1604, 24 hrs post-transfection cells were treated with IL-4 or left untreated and luciferase activity was measured after 24 hrs of IL-4 treatment. Results are mean \pm S.D. of three individual experiments. *, $\mathrm{P}<0.05 ; * *, \mathrm{P}<0.01$.

Figure 6. IL-4-induced expression of CYP2E1 is mediated through the activation of NFATc1. (A) mRNA levels of the transcription factors NF-кB, Elk1, c-fos, c-jun, Sp1, NFATc1, NFAT4 and FOXO1 were measured in IL-4 treated or untreated B16A2 cells. Results are expressed as the fold induction of IL-4 treated over untreated control cells and normalized against $\beta$-actin. (B) siRNA expression constructs targeting the transcription factors NF-kB, Elk1, NFATc1 and FOXO1 were co-transfected together with the CYP2E1 promoter construct 2E1-1604. Twenty-four hours post-transfection cells were treated with IL-4 and luciferase activity was measured after an additional 24 hrs. (C) The CYP2E1 promoter construct 2E1-1604 was co-transfected with expression constructs for NFATc1 or FOXO1 and luciferase activity was determined 48 hours later. Results are mean \pm S.D. of three individual experiments. *, P $<0.05$. (D). Interaction of NFATc1 with the CYP2E1 promoter as determined by chromatin immunoprecipitation (ChIP) analysis. Confluent B16A2 cells transfected with 2E1-2651were treated with IL4 or left untreated and DNA-protein complexes were immunoprecipitated with a specific NFATc1 antibody or as a control a non-relevant IgG. Input DNA is an aliquot of the sheered DNA prior to immunoprecipitation.

Figure 7. Both STAT6 and NFATc1 are required for efficient IL-4 induction of CYP2E1. Mutations in the STAT6 and NFATc1 responsive elements were introduced in the CYP2E1 promoter construct 2E1-1604, resulting in $\triangle$ STAT6 (mutation of the 
proximal STAT6 responsive element only), $\triangle$ NFATc1 (mutation of the distal NFATc1 responsive element only) and $\Delta \mathrm{STAT} 6+\Delta \mathrm{NFATc} 1$ (mutation of both STAT6 and NFATC1 responsive elements). siRNA expression constructs targeting STAT6 (siSTAT6) and NFATc1 (siNAFTc1) were co-transfected with the wtCYP2E1 promoter construct 2E1-1604, 24 hours post-transfection cells were treated with IL-4 and luciferase activity was measured. The position of the STAT6 and NFATc1 responsive elements in the human CYP2E1 are schematically shown at the bottom of the figure together with the mutated residues (shown in black box). Results are mean \pm S.D. of three individual experiments. *, $\mathrm{P}<0.05$, **, $\mathrm{P}<0.01$ as compared to IL-4 treated cells.

Figure 8. Diagram of IL-4 mediated CYP2E1 gene transcription. The factors that were identified in the present study to be important for IL-4 induced CYP2E1 expression are indicated with grey arrows. STAT6-BE: STAT6 binding element; NFATc1-BE:

NFATc1 binding element. See text for further details. 


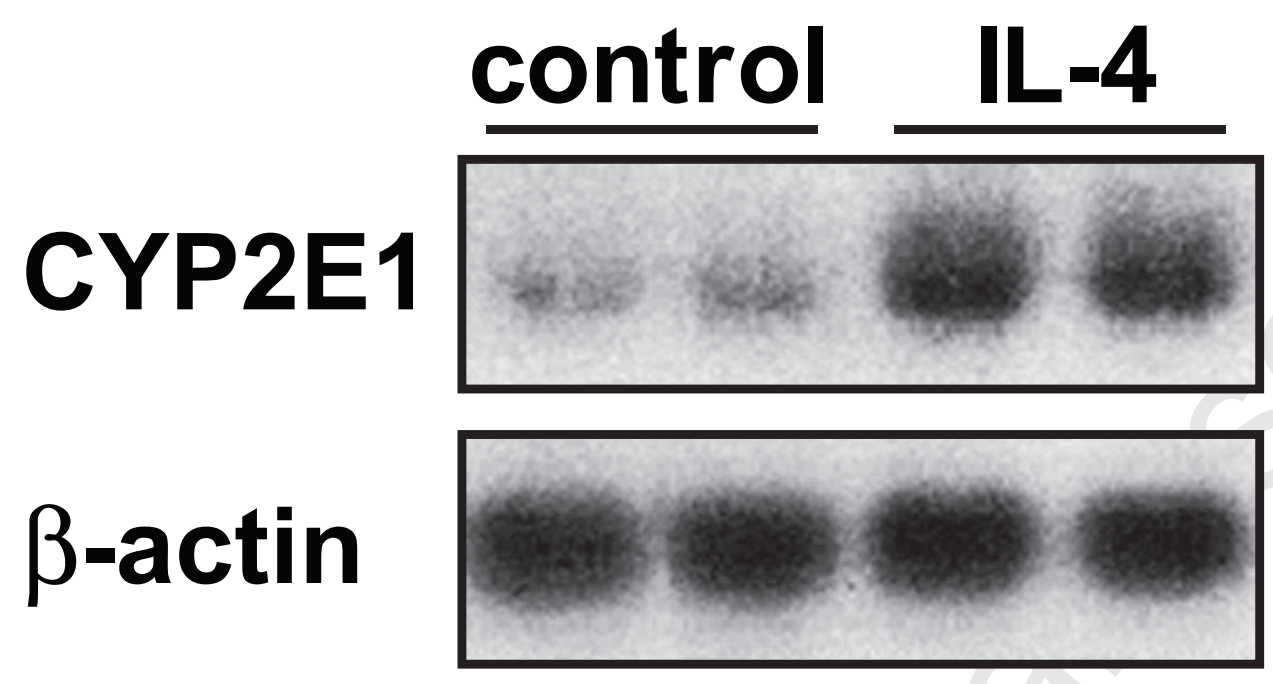

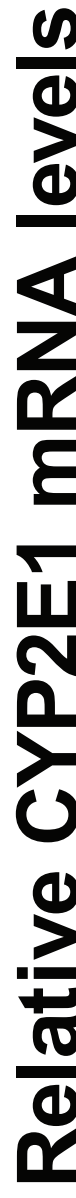

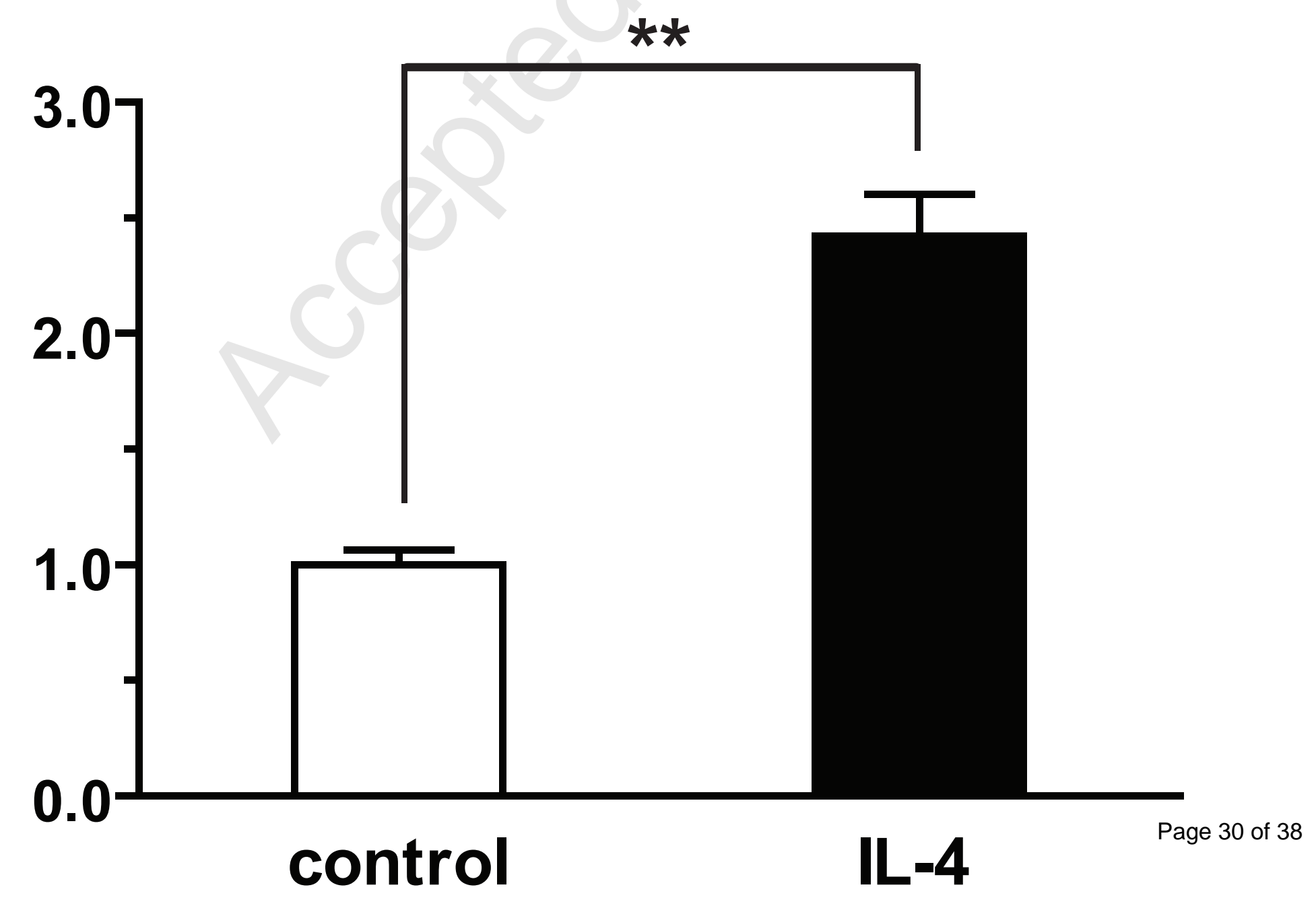




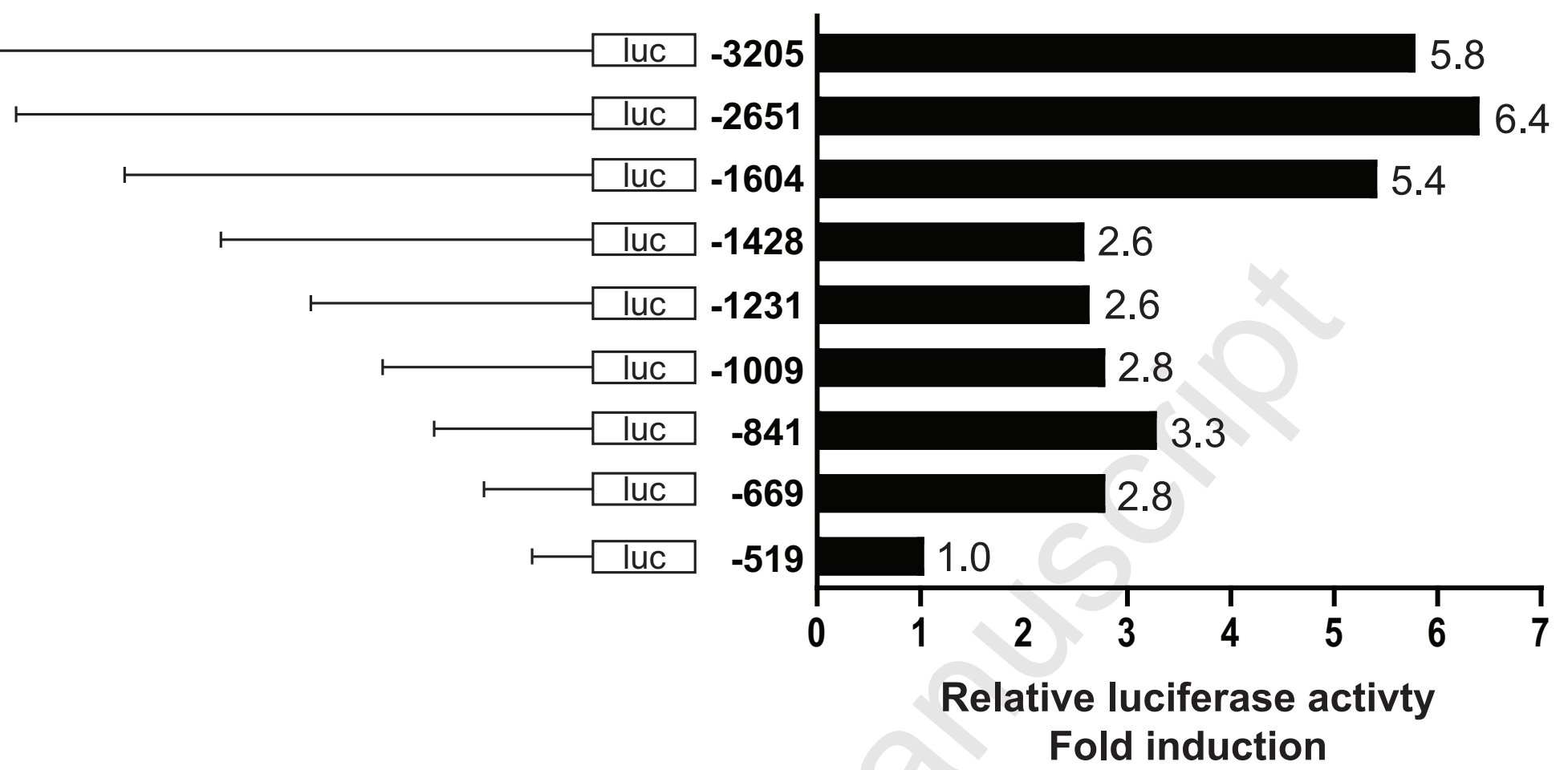


Figure 4

A

B
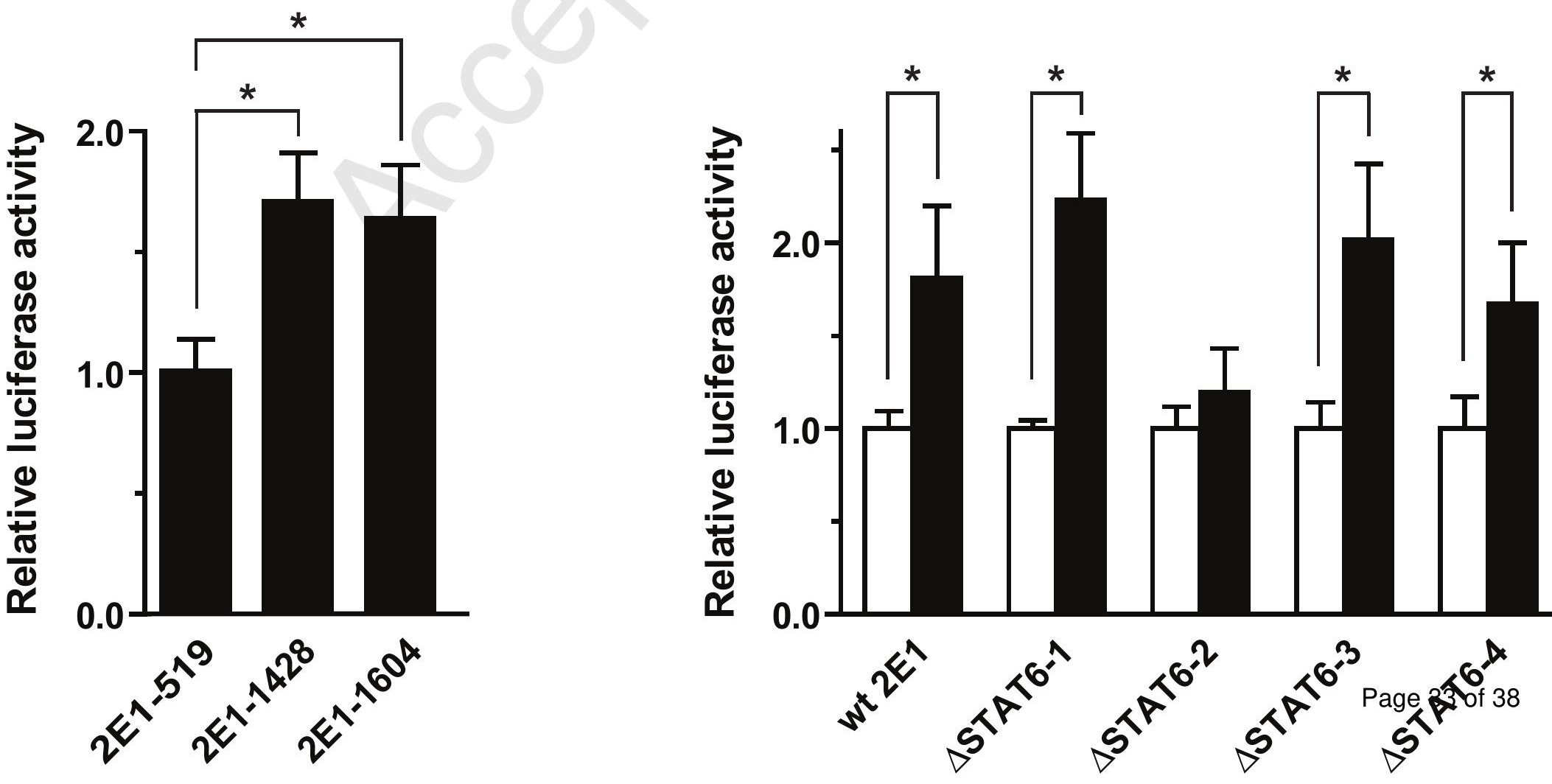
Figure 5

A

$\begin{array}{ll}\text { IRS1 IRS2 } & \text { IR1 }\end{array}$



B 


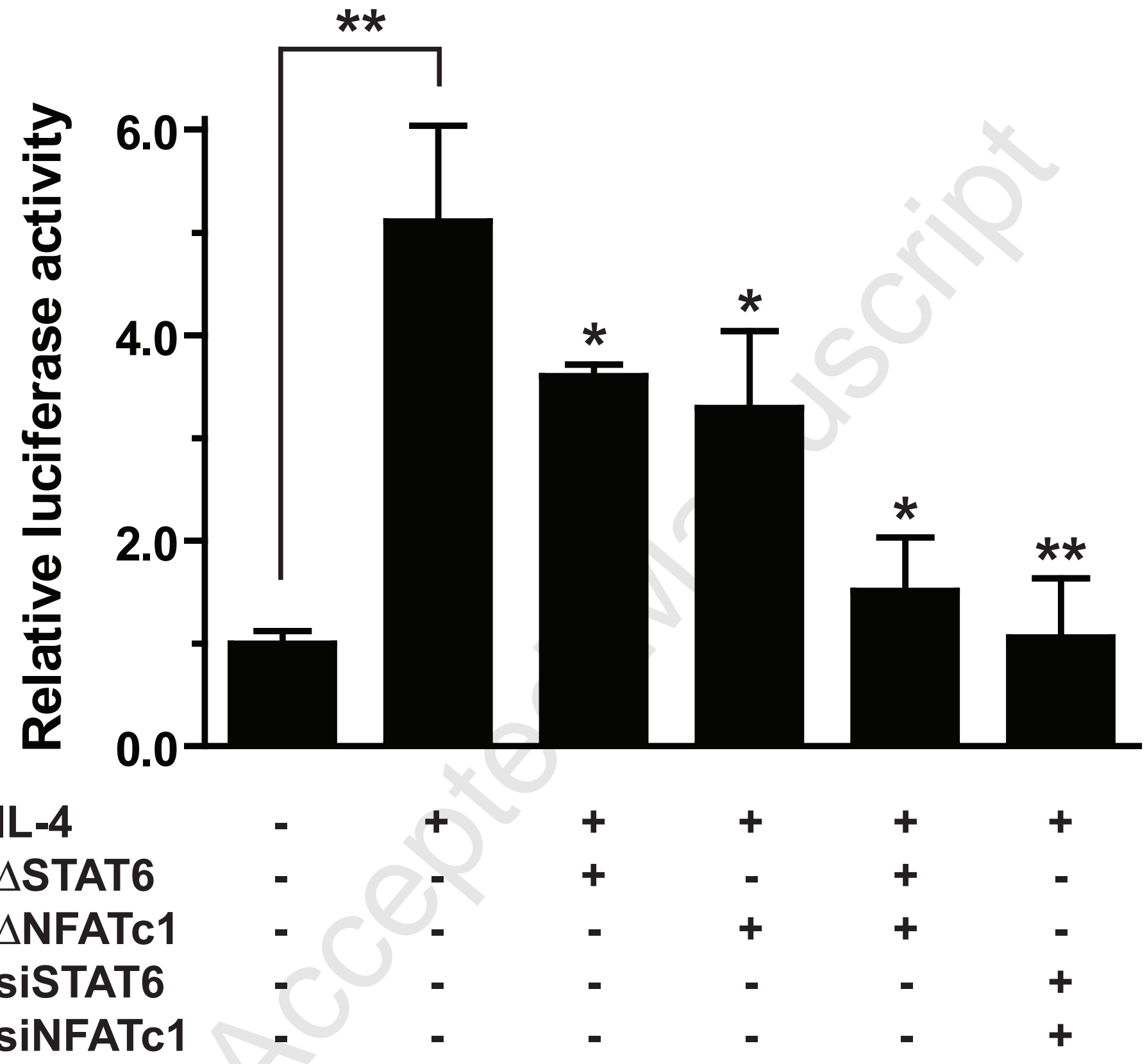

aGGAAAC

TTCcatgGAA

TTCAAAC

$\Delta$ NFATc 1
AAGcatgGAA $\triangle$ STAT6 


\section{Figure 8}
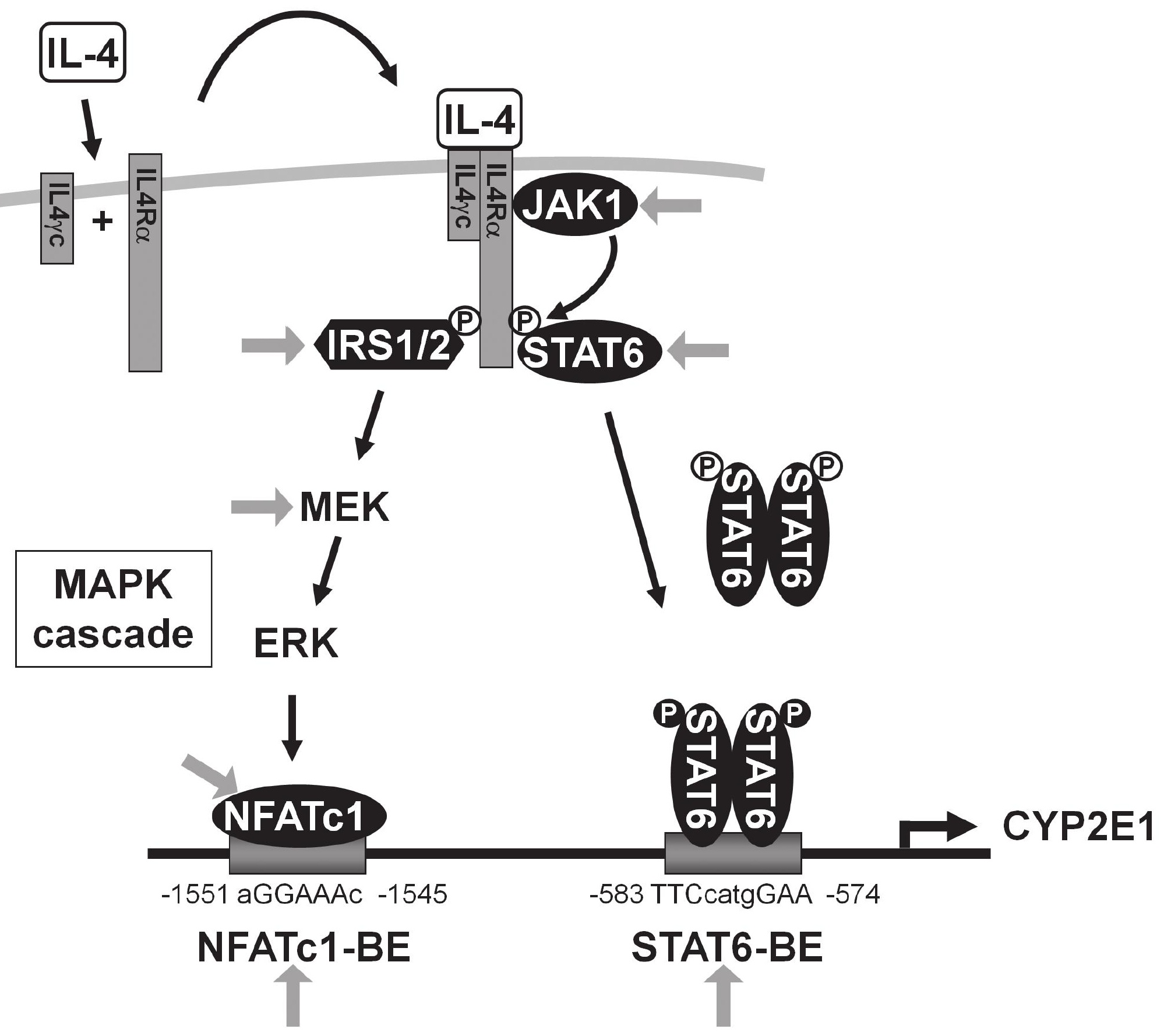


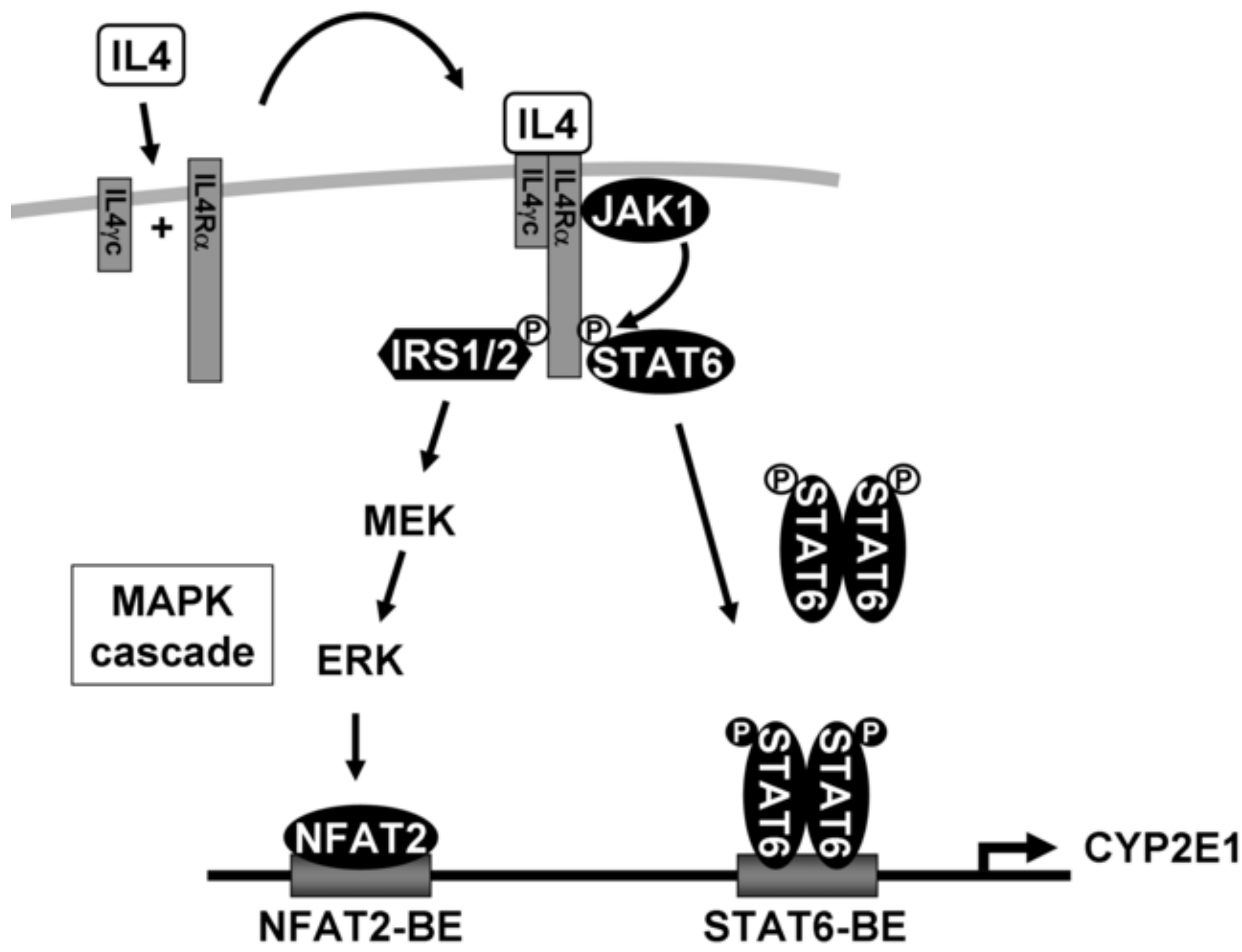

